Radial and Nonradial Pulsations as Probes of Stellar Physics

ASP Conference Series, Vol. 259, 2002

C. Aerts, T.R. Bedding, \& J. Christensen-Dalsgaard, eds.

\title{
A New Way to Resolve Cepheid Binaries ${ }^{1}$
}

\author{
Nancy Remage Evans \\ Smithsonian Astrophysical Observatory, Cambridge, MA, USA
}

Derck Massa

Emergent IT, MD, USA

\begin{abstract}
We have measured the centroid shift of low resolution HST FOC spectra as the dominant star changes from the Cepheid to the hot companion. With this approach we have resolved the AW Per system and marginally resolved the U Aql system.
\end{abstract}

\section{Introduction}

Spatially resolving Cepheid binary systems is the first step in measuring the inclination of the orbit. A successful resolution means that ultimately both the mass and distance can be determined from first principles. This provides cosmologically important distances, and also benchmarks for stellar evolution calculations.

\section{Observations}

We have developed a new approach to resolving Cepheid binaries using the Hubble Space Telescope Faint Object Camera (HST FOC) in the objective prism mode. The basic approach was first put forward by Massa \& Endal $(1987 a, b)$. In Cepheid binary systems, bright companions will be hot main sequence stars. In this case, the Cepheid dominates in the visible part of the spectrum, however it contributes very little flux to the ultraviolet spectrum, where the hot companion dominates. The two components may be unresolved in an image. In the low resolution FOC spectra, however, the location of the centroid of the spectrum on the detector will shift as a function of wavelength as the star producing the spectrum changes from the Cepheid in the visible to the companion in the ultraviolet. The locations of the spectrum centroids for the two stars can be measured very accurately, better than 0.01 " for the FOC. We have now obtained first epoch observations of both AW Per and U Aql with the HST FOC (Faint Object Camera) with the NUV (Near Ultraviolet) objective prism.

\footnotetext{
${ }^{1}$ Based on observations made with the NASA/ESA Hubble Space Telescope, obtained at the Space Telescope Science Institute, which is operated by the Association of Universities for Research in Astronomy, Inc. under NASA Contract No. NASA-26555
} 


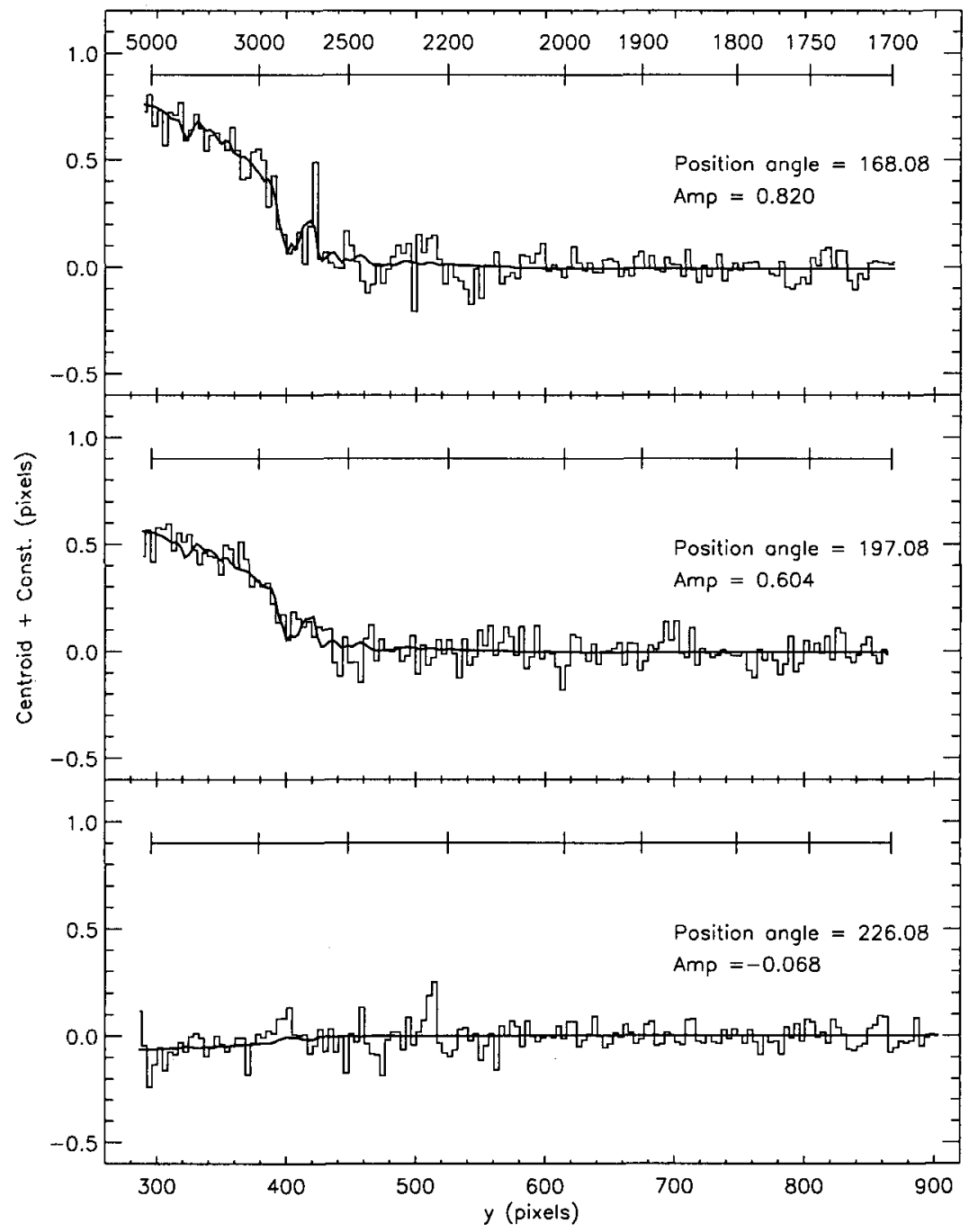

Figure 1. The location of the centroid of the AW Per spectra shown for 3 roll angles (specified on the right) in the 3 panels. Both coordinates are in pixels, but the wavelength scale is given at the top of the figure. The data are shown as histograms; the solid line is the model derived from the flux distribution. 


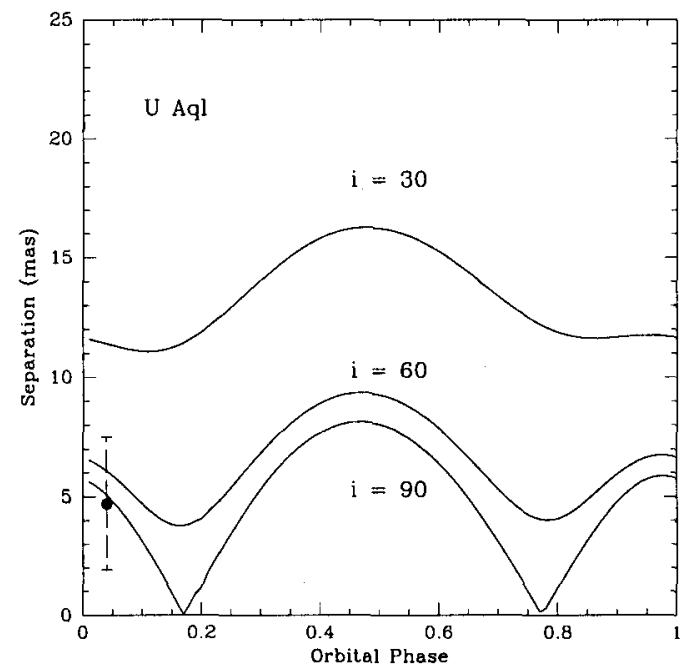

Figure 2. The predicted separation between the Cepheid U Aql A and the companion (in mass) as a function of orbital phase from the ground-based spectroscopic orbit of the Cepheid. Three values of the inclination are illustrated. The point shows the value of the separation measured with the FOC.

\section{Results}

Measuring the inclination of a system requires two observations at phases carefully selected from the ground-based orbit of the Cepheid. In addition, at each epoch, several observations must be made with varying satellite roll angles to determine the magnitude and orientation of the angular separation on the sky.

Results are shown in Fig. 1. The data are fitted to a model of the relative contribution of each component based on observed IUE spectra of the composite system, which, of course, includes the reddening (Evans, 1992, 1994). (Residual distortions in the field have been removed with a fourth order polynomial.) The three values of the projected separation ("Amp" in Fig. 1), are then fitted as a function of the roll angle to determine the full separation.

Using this approach, we have obtained a clear resolution of the Cepheid AW Per at one epoch (separation $0.013 \pm 0.003 "$ ). We also have a marginal resolution of U Aql.

What can we say about the results so far? Fig. 2 is an example using U Aql, showing the predicted separations for three inclinations from the spectroscopic orbit of the Cepheid (Welch et al., 1987). The mass ratio between the Cepheid and the companion is taken from this orbit and the velocity of the companion measured with the HST Goddard High Resolution Spectrograph (Evans et al., 1998). While a second centroid measurement is required to determine the 
inclination independently without using a mass ratio, Fig. 2 shows that combination of the separation already measured and the mass ratio requires a high inclination. Full results will be given in Massa \& Evans (2002).

\section{Future work}

Observations for the second epoch are being scheduled on the HST STIS spectrograph. STIS should provide improved accuracy because of easier extraction from geometrically distinct pixels, and higher photometric sensitivity.

\section{References}

Evans, N. R. 1992, ApJ, 389, 657

Evans, N. R. 1994, ApJ, 436, 273

Evans, N. R., Böhm-Vitense, E., Carpenter, K., Beck-Winchatz, B., \& Robinson, R. 1998, ApJ, 494, 768

Massa, D. \& Endal, A. S. 1987a, AJ, 93579

Massa, D. \& Endal, A. S. 1987b, AJ, 93760

Massa, D. \& Evans, N. R. 2002, in preparation

Welch, D. L., Evans, N. R., Lyons, R. W., Harris, H. C., Barnes, T. G. III, Slovak, M. H., \& Moffett, T. J. 1987, PASP, 99, 610

\section{Discussion}

M. Jerzykiewicz : How sensitive is your method to interstellar reddening?

$N$. Evans : The model to which the centroid shift is compared is created from the observed IUE composite spectrum so it already includes reddening. In addition, each star completely dominates at one end of the spectrum, so fluxes of each star are well determined.

T. Bedding : Comment: The idea of using centroid variations as a function of wavelength from spectra has also been developed by Jeremy Bailey from AAO who calls it "spectro-astrometry" (e.g. Bailey, 1998, MNRAS, 301, 161). Question: There seems to be a scatter in your luminosity-mass relation (e.g. Fig. 5 in Evans et al., 1998). Could this be due to rotation as an extra parameter?

N. Evans : Rotation is certainly a possibility. At this point, I'm looking forward to reducing the error bars enough to be able to make strong statements about something like that. 


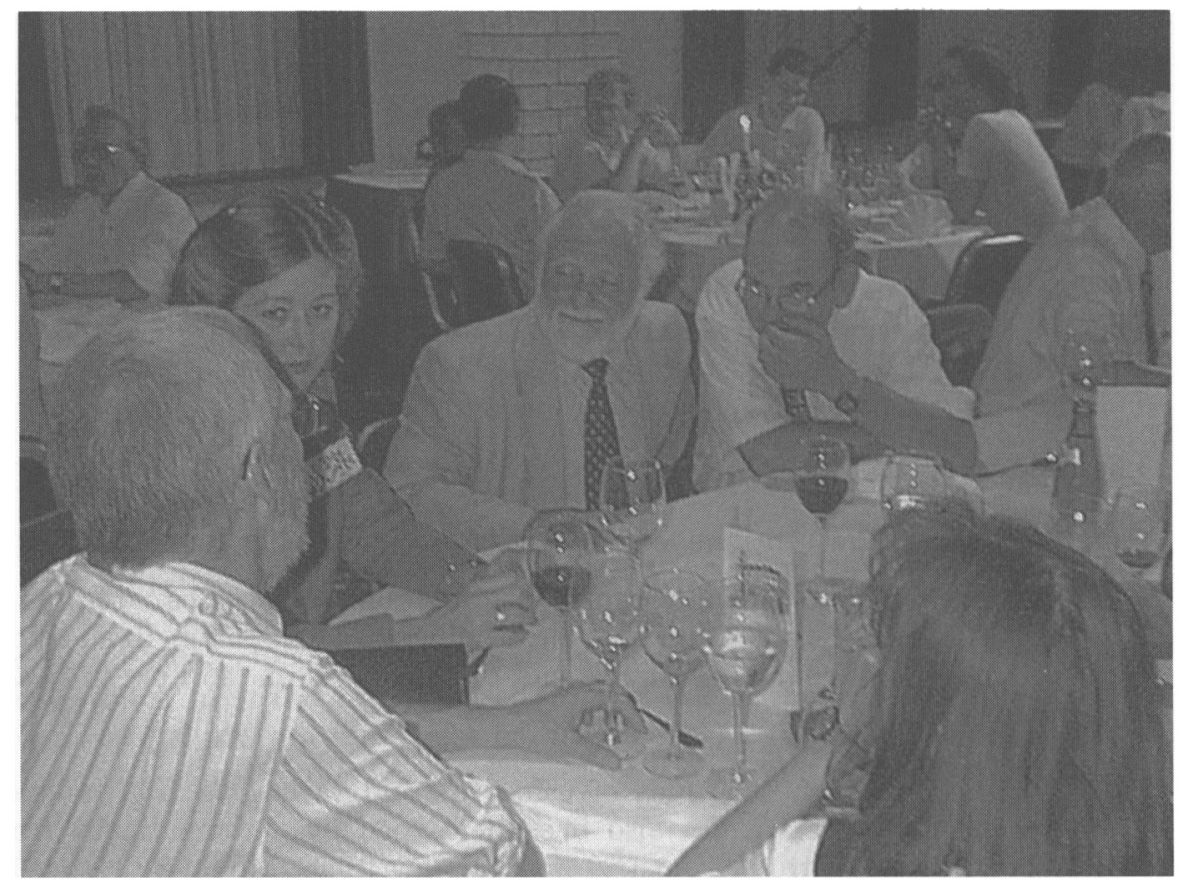

Don Kurtz just told a joke. Wojtek Dziembowski obviously liked it, but it seems that Douglas Gough didn't quite understand the point. June Kurtz feels a bit embarrassed. 